\title{
PGC1a protects against cisplatin-induced skeletal muscle dysfunction
}

\author{
Rohit Chatterjee ${ }^{1}$, Joshua Huot ${ }^{2}$, Fabrizio $\mathrm{Pin}^{2}$, Andrea Bonetto ${ }^{2}$ \\ ${ }^{1}$ Indiana University School of Medicine; ${ }^{2}$ Indiana University School of Medicine, Department of \\ Surgery
}

\section{Background and Hypothesis:}

We and others have shown that chemotherapy promotes skeletal muscle wasting and weakness (i.e., cachexia) by disrupting mitochondrial homeostasis and causing oxidative stress. Peroxisome proliferative-activated receptor gamma coactivator 1-alpha (PGC1 $\alpha$ ) is a pivotal regulator of mitochondrial biogenesis and is involved in reducing oxidative damage in skeletal muscle. Hence, in the present study we investigated whether overexpression of skeletal muscle PGC1 $\alpha$ (mPGC1 $\alpha$ ) was sufficient to preserve skeletal muscle mass and function in young and old mice treated with cisplatin.

\section{Experimental Design or Project Methods:}

Young (2-month; $n=5$ ) and old (18-month; $n=5-8$ ) male wild type (WT) or mPGC1a transgenic mice were treated with cisplatin $(2.5 \mathrm{mg} / \mathrm{kg})$, while age-matched WT mice received vehicle for 2 weeks. Animals were assessed for muscle force and motor unit number estimation (MUNE). Skeletal muscles were weighed and processed for molecular analyses, including assessment of mitochondrial protein content.

\section{Results:}

Young WT mice exposed to cisplatin showed evidence of cachexia, as indicated by reduced gastrocnemius size (-16\%), plantarflexion force $(-8 \%)$ and MUNE $(-56 \%)$, whereas mPGC1 $\alpha$ mice were only partially protected. Interestingly, despite exacerbated cachexia in aged WT mice treated with chemotherapy, as demonstrated by markedly decreased gastrocnemius size ($22 \%)$, plantarflexion force $(-18 \%)$ and MUNE $(-80 \%)$ compared to untreated WT, muscle mass, strength and innervation were fully preserved in age-matched mPGC1a mice. Follow-up molecular analyses revealed that WT animals exposed to chemotherapy present loss of muscle mitochondrial proteins PGC1 $\alpha$, OPA1 and CytochromeC, whereas their levels in mPGC1 $\alpha$ mice were robustly increased.

\section{Conclusion and Potential Impact:}

Altogether, our data suggest that PGC1a plays a pivotal role in preserving skeletal muscle mass and function, usually impaired by anticancer treatments. These findings enforce developing mitochondria-targeting therapeutics to combat the negative consequences that chemotherapy has on skeletal muscle. 